\title{
INSTITUT ROYAL DU PATRIMOINE ARTISTIQUE RADIOCARBON DATES III
}

\author{
M. DAUCHOT-DEHON and J. HEYLEN \\ Institut Royal du Patrimoine artistique, Brussels, Belgium
}

INTRODUCTION

This list includes the results of datings made during 1969-70. The method is essentially the same as described in R., 1968, v. 10, p. 29-35; 1971 , v. 13, p. 29-31.

\section{SAMPLE DESCRIPTIONS}

I. GEOLOGIC SAMPLES

\section{IRPA-66. St. Joris ten Distel}

Peat from St. Joris ten Distel, W Flanders, Belgium $\left(51^{\circ} 07^{\prime} 40^{\prime \prime} \mathrm{N}\right.$ Lat, 3० 19' 50" E Long), at $10 \mathrm{~m}$ alt. Coll. 1969 by W. De Breuck, Lab. Geol., Univ. of Ghent, Belgium. No NaOH pretreatment. Comment: control of palynologic dates.

\section{IRPA-67. St. Baafs Vijve}

Shells from St. Baafs Vijve, W Flanders, Belgium $\left(50^{\circ} 54^{\prime} 40^{\prime \prime} \mathrm{N}\right.$ Lat, $3{ }^{\circ} 23^{\prime} 45^{\prime \prime}$ E Long), at $10 \mathrm{~m}$ alt. Coll. 1969 by G. De Moor, Lab. Geol., Univ. of Ghent, Belgium; subm. by W. De Breuck. Pretreatment with $\mathrm{HCl} 1 \%$ until 15 to $20 \%$ weight loss. Comment: date elucidates unconfirmed data.

\section{IRPA-68. St. Baafs Vijve}

Peat from St. Baafs Vijve, W Flanders, Belgium $\left(50^{\circ} 54^{\prime} 40^{\prime \prime} \mathrm{N}\right.$ Lat, $3^{\circ} 23^{\prime} 45^{\prime \prime} \mathrm{E}$ Long), at $1.5 \mathrm{~m}$ alt. Coll. by G. De Moor; subm. by W. De Breuck. No NaOH pretreatment. Comment: sample was taken from peat layer, at 7 to $8 \mathrm{~m}$ depth, between sablon upper layer and loam under layer.

\section{IRPA-69. Zandvoorde}

$3365 \pm 190$

1415 B.c.

Peat from Zandvoorde, W Flanders, Belgium $\left(51^{\circ} 12^{\prime} 30^{\prime \prime} \mathrm{N}\right.$ Lat, $2^{\circ} 57^{\prime} 30^{\prime \prime} \mathrm{E}$ Long), at $1.8 \mathrm{~m}$ alt and $15 \mathrm{~cm}$ depth. Coll. 1969 by G. De Moor; subm. by W. De Breuck. No NaOH pretreatment. Comment: sample belongs to IRPA-39-42 series, R., 1971, v. 13, p. 29. See general comment to series.

\section{Meetkerke series}

Samples from W Flanders, Belgium (51 $13^{\prime} 32^{\prime \prime}$ N Lat, $3^{\circ} 9^{\prime} 43^{\prime \prime}$ E Long). Coll. 1969 to 1970 by W. De Breuck and G. De Moor. 


\section{IRPA-76. Meetkerke 1}

Shells (Scrobicularia), at $-5 \mathrm{~m}$ alt; pretreated with $\mathrm{HCl} 1 \%$ until 15 to $20 \%$ weight loss.

\section{IRPA-77. Meetkerke 2}

$21,440 \pm 670$

Shells (Ostrea), at $-5 \mathrm{~m}$ alt; pretreated as above.

\section{IRPA-78. Meetkerke 3}

$17,430 \pm 460$

Shells (Hydrobia), at $-4 \mathrm{~m}$; pretreated as above.

IRPA-79. Meetkerke 4

Shells (Cardium), at $0 \mathrm{~m}$ alt; pretreated as above.

$$
19,490 \text { в.C. }
$$

15,480 в.C.

$22,875 \pm 620$ 20,925 в.C.

\section{IRPA-86. Meetkerke 5}

$34,160 \pm 850$

32,210 в.c.

Shells (Cardium, Venerupis, Nasso, Scrobicularia), at $-5 \mathrm{~m}$ alt; pretreated as above.

IRPA-85. St. Pieters Brugge

$>35,000$

Wood of calcined trunk, at $-3 \mathrm{~m}$ alt. $\left(51^{\circ} 13^{\prime} 32^{\prime \prime} \mathrm{N}\right.$ Lat, $3^{\circ} 11^{\prime}$ $38^{\prime \prime}$ E Long).

General Comment (W. De B.): dates are used for stratigraphic study of upper Pleistocene in coastal area. Samples were from sediments and were not handled again.

\section{Pajara series}

Calcareous rock from Pajara, Canary Is., Spain $\left(28^{\circ} 24^{\prime} \mathrm{N}\right.$ Lat, $10^{\circ}$ $27^{\prime} \mathrm{W}$ Long), at $75 \mathrm{~m}$ alt. Coll. 1969 by P. De Paepe and R. Vermeire, Univ. of Ghent, Belgium; subm. by W. De Breuck. Pretreatment with HCl $1 \%$ until 15 to $20 \%$ weight loss.

\section{IRPA-80. Pajara 1}

$25,850 \pm 560$

\section{IRPA-81. Pajara 2} 23,900 в.C.

$$
\begin{aligned}
& \mathbf{7 0 8 0} \pm \mathbf{3 0 0} \\
& \mathbf{5 1 3 0} \text { в.C. }
\end{aligned}
$$

\section{IRPA-84. Pajara 3}

$19,405 \pm 540$ 17,455 в.C.

General Comment (P. De P.): dates beginning (IRPA-80) and end (IRPA81) of calcareous rock formation. IRPA-84 is sample of intermediate layer.

\section{IRPA-87. Uitkerke}

$$
1630 \pm 90
$$

Shells (Cardium) from Uitkerke, W Flanders, Belgium (51 $11^{\prime} 35^{\prime \prime}$ $\mathrm{N}$ Lat, $1^{\circ}$ 13' 13" E Long), at $0 \mathrm{~m}$ alt. Coll. 1969 by W. De Breuck. Pretreatment $\mathrm{HCl} 1 \%$ until 15 to $20 \%$ weight loss. Comment: shells from well at $2.5 \mathrm{~m}$ depth; date confirms previous data. 
Institut Royal Du Patrimoine Artistique Radiocarbon Dates III 147

IPJA-88. Izenberge

Shells (Cardium) from Izenberge, W Flanders, Belgium $\left(50^{\circ} 1^{\prime} 18^{\prime \prime}\right.$ $\mathrm{N}$ Lat, $2^{\circ} 43^{\prime} 36^{\prime \prime} \mathrm{E}$ Long), at $11 \mathrm{~m}$ alt. Coll. 1970 by M. De Dapper, Lab. Geol., Univ. of Ghent, Belgium. Pretreatment HCl $1 \%$ until 15 to $20 \%$ weight loss.

IRPA-89. Ghent

$30,005 \pm 735$ 28,055 B.c.

Shells from Ghent, E Flanders, Belgium $\left(51^{\circ} 06^{\prime} 05^{\prime \prime} \mathrm{N}\right.$ Lat, $3^{\circ}$ $45^{\prime} 30^{\prime \prime}$ E Long), at $-10 \mathrm{~m}$ alt. Coll. 1965 by G. De Moor. Pretreated as above.

\section{Weelde series}

\section{ARCHAEOLOGIC SAMPLES}

Charcoal from Weelde excavations, Flanders, Belgium $\left(51^{\circ} 25^{\prime} 18^{\prime \prime}\right.$ N Lat, 5 2' 12" E Long). Coll. 1965 by H. Roosens, Service Nat. des Fouilles, Brussels, Belgium. Comment (H.R.): dates agree with archaeologic date: Bronze age.

IRPA-49. Weelde 1

$2880 \pm 130$

930 B.C.

IRPA-51. Weelde 2

$2915 \pm 160$

965 B.C.

IRPA-52. Weelde 3

$3155 \pm 160$

1205 B.c.

\section{Jemdet Nasr series}

Coal from Jemdet Nasr; Mesopotamia $\left(32^{\circ} 24^{\prime} \mathrm{N}\right.$ Lat, $44^{\circ} 43^{\prime}$ E Long). Coll. 1967 by L. De Meyer, Univ. of Ghent, Belgium. Comment (L. De M.): before excavation, level was $12 \mathrm{~m}$ above ground level.

\section{IRPA-70. Jemdet A}

$4470 \pm 220$

Coal near mud brick wall, $1 \mathrm{~m}$ under present level. Archaeologic date: ca. 2700 B.c.

\section{IRPA-71. Jemdet B}

$3640 \pm 175$

Coal among ashes and burned bones, at $40 \mathrm{~cm}$ ur Also found Also found was a painted vase dated at end of archaic dynasty or beginning of Agade age: ca. 2400 в.c.

IRPA-72. Jemdet $C$

$$
2140 \pm 130
$$

Coal from same site as IRPA-71, tamination. 
IRPA-73. Jemdet D

Coal from filling of ground and stones near a brick pipe, at $60 \mathrm{~cm}$ under present level. Filling is probably contemporary with pipe: ca. 2400 B.C.

IRPA-74. Jemdet E

$3470 \pm 190$ 1520 B.c.

Coal from brick well, between $1.5 \mathrm{~m}$ and $1.6 \mathrm{~m}$ of present margin, with ceramics dated at end of Ur III age or beginning of Isin-Larsa age: ca. 2000 B.c.

IRPA-75. Jemdet F

$3830 \pm 215$

Coal from ground, near oven, at $50 \mathrm{~cm}$ under present level.

\section{REFERENCE}

Dauchot-Dehon, M. and Heylen, J., 1971, Institut royal du Patrimoine artistique radiocarbon dates II: Radiocarbon, v. 13, p. 23-31. 